\title{
3 \\ Compression of Digital Biomedical Signals
}

\section{A. Enis Çetin Hayrettin Köymen Bilkent University}

3.1 Introduction.

3.2 Time-Domain Coding of Biomedical Signals.......... 3-2

Data Compression by DPCM • AZTEC ECG Compression

Method • Turning Point ECG Compression Method • ECG Compression via Parameter Extraction

3.3 Frequency-Domain Data Compression Methods .....
Wavelet or Subband Coding

Hybrid Multichannel ECG Coding

Preprocessor • Linear Transformer • Compression of the

Transform Domain Signals • Subband Coder (SBC)

3.6 Conclusion .................................. 3-11

References ...................................... 3-11

Further Information .............................. 3-12

\subsection{Introduction}

Computerized electrocardiogram (ECG), electroencephalogram (EEG), and magnetoencephalogram (MEG) processing systems have been widely used in clinical practice [1] and they are capable of recording and processing long records of biomedical signals. The use of such systems (1) enables the construction of large signal databases for subsequent evaluation and comparison, (2) makes the transmission of biomedical information feasible over telecommunication networks in real time or off line, and (3) increases the capabilities of ambulatory recording systems such as the Holter recorders for ECG signals. In spite of the great advances in VLSI memory technology, the amount of data generated by digital systems may become excessive quickly. For example, a Holter recorder needs more than $200 \mathrm{Mbits} /$ day of memory space to store a dual-channel ECG signal sampled at a rate of 200 samples/sec with $10 \mathrm{bit} / \mathrm{sample}$ resolution. Since the recorded data samples are correlated with each other, there is an inherent redundancy in most biomedical signals. This can be exploited by the use of data compression techniques which have been successfully utilized in speech, image, and video signals [2] as well.

The aim of any biomedical signal compression scheme is to minimize the storage space without losing any clinically significant information, which can be achieved by eliminating redundancies in the signal, in a reasonable manner. 
Data compression methods can be classified into two categories: (1) lossless and (2) lossy coding methods. In lossless data compression, the signal samples are considered to be realizations of a random variable or a random process and the entropy of the source signal determines the lowest compression ratio that can be achieved. In lossless coding the original signal can be perfectly reconstructed. For typical biomedical signals lossless (reversible) compression methods can only achieve Compression Ratios (CR) in the order of 2 to 1 . On the other hand lossy (irreversible) techniques may produce CR results in the order of 10 to 1 . In lossy methods, there is some kind of quantization of the input data which leads to higher CR results at the expense of reversibility. But this may be acceptable as long as no clinically significant degradation is introduced to the encoded signal. The CR levels of 2 to 1 are too low for most practical applications. Therefore, lossy coding methods which introduce small reconstruction errors are preferred in practice. In this section we review the lossy biomedical data compression methods.

\subsection{Time-Domain Coding of Biomedical Signals}

Biomedical signals can be compressed in time domain, frequency domain, or time-frequency domain. In this section the time domain techniques, which are the earlier approaches to biomedical signal compression, are reviewed.

\subsubsection{Data Compression by DPCM}

Differential pulse code modulation (DPCM) is a well-known data coding technique in which the main idea is to decorrelate the input signal samples by linear prediction. The current signal sample, $x(n)$, is estimated from the past samples by using either a fixed or adaptive linear predictor

$$
\hat{x}(n)=\sum_{k=1}^{N} a_{k} x(n-k)
$$

where $\hat{x}(n)$ is the estimate of $x(n)$ at discrete time instant $n$, and $\left\{a_{k}\right\}$ is the predictor weight. The samples of the estimation error sequence, $e(n)=x(n)-\hat{x}(n)$ are less correlated with each other compared to the original signal, $x(n)$ as the predictor removes the unnecessary information which is the predictable portion of the sample $x(n)$. In a typical DPCM encoder, the error sequence is quantized by using a nonuniform quantizer and quantizer outputs are entropy coded by assigning variable-length codewords to the quantized error sequence according to the frequency of occurrence. The variable-length codebook is constructed by Huffman coding which assigns shorter (longer) codewords to values occurring with higher (lower) probabilities. Huffman coding produces compression results that are arbitrarily close to the entropy of the quantized error sequence.

A CR of 7.8 was reported for an ECG signal recorded at a rate of $500 \mathrm{~Hz}$ with 8 bit/sample resolution [3]. This means that about $1 \mathrm{bit} / \mathrm{sample}$ is used to represent the ECG signal. The corresponding percent root mean square difference (PRD) was 3.5. The PRD is a measure of reconstruction error and it is defined as follows:

$$
\mathrm{PRD}=\sqrt{\frac{\sum_{n=0}^{N-1}\left[x(n)-x_{\mathrm{rec}}(n)\right]^{2}}{\sum_{n=0}^{N-1} x^{2}(n)}} * 100
$$

where $N$ is the total number of samples in the ECG signal, $x(n)$, and $x_{\text {rec }}(n)$ is the reconstructed ECG signal.

A typical DPCM encoder may become a lossless coder if the quantization step is skipped. In this case CR drops drastically to low values. 


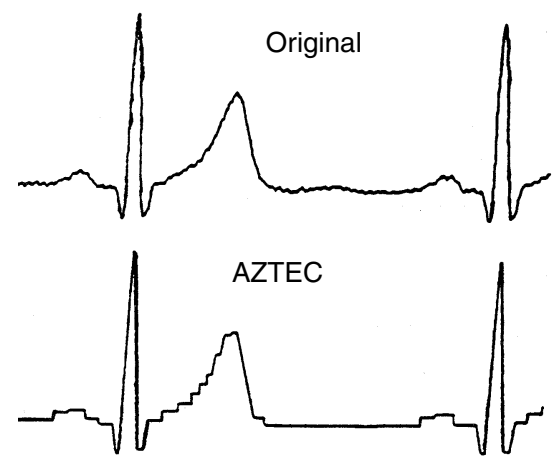

FIGURE 3.1 AZTEC representation of an ECG waveform.

\subsubsection{AZTEC ECG Compression Method}

The Amplitude Zone Time Epoch Coding (AZTEC) is one of the earliest ECG coding methods. It was developed by Cox et al. [4] as a preprocessing software for real-time monitoring of ECGs. It was observed to be useful for automatic analysis of ECGs such as QRS detection, but it is inadequate for visual presentation of the ECG signal as the reconstructed signal has a staircase appearance.

In this method the ECG signal is considered to consist of flat regions and "slopes." If the signal value stays within a predetermined constant for more than three consecutive samples then that region is assumed to be constant and stored by its duration (number of samples) and a fixed amplitude value. Otherwise the signal is assumed to have a slope. A slope is stored by its duration and the amplitude of the last sample point. Linear interpolation is used to reconstruct the ECG signal. As a result, the resulting signal has a discontinuous nature as shown in Figure 3.1.

Even through AZTEC produces a high compression ratio (CR $=10$ to 1 , for $500 \mathrm{~Hz}$ sampled data with $12 \mathrm{bit} / \mathrm{sample}$ resolution) the quality of the reconstructed signal is very low and it is not acceptable to the cardiologists.

Various modified of AZTEC are proposed [5]. One notable example is the CORTES technique [6]. The CORTES technique is a hybrid of the AZTEC and the Turning Point method which is described in the next section.

\subsubsection{Turning Point ECG Compression Method}

The Turning Point data reduction method [7] is basically an adaptive downsampling method developed especially for ECGs. It reduces the sampling frequency of an ECG signal by a factor of two.

The method is based on the trends of the signal samples. Three input samples are processed at a time. Let $x(n)$ be the current sample at discrete-time instant $n$. Among the two consecutive input samples, $x(n+1)$ and $x(n+2)$, the one producing the highest slope (in magnitude) is retained and the other sample is dropped. In this way the overall sampling rate is reduced to one-half of the original sampling rate; no other coding is carried out. Therefore, the resulting CR is 2 to 1 . A PRD of $5.3 \%$ is reported for an ECG signal sampled at $200 \mathrm{~Hz}$ with $12 \mathrm{bit} /$ sample resolution [7]. In practice the CR value actually may be lower than two as the retained samples may not be equally spaced and some extra bits may be needed for timing determination.

\subsubsection{ECG Compression via Parameter Extraction}

In these methods, the signal is analyzed and some important features such as typical cycles, extreme locations, etc. are determined. These features are properly stored. Reconstruction is carried out by using appropriate interpolation schemes. 
The location of the extrema or peaks in an ECG signal is important in diagnosis because they basically determine the shape of each ECG period. The ECG compression techniques described in Reference 8 take advantage of this feature and record only the maxima, minima, slope changes, zero-crossing intervals, etc. of the signal. During reconstruction various interpolation schemes such as polynomial fitting and spline functions are used. The performance of the extrema based methods is compared to the AZTEC method and it was reported that for a given CR the RMS error is half of that of AZTEC method [8].

Other parameter extraction methods include $[9,10]$ where ECG signals are analyzed in a cyclesynchronous manner. An AR model is fitted to the MEG signal and parameters of the AR model are stored [11]. An ECG signal is modeled by splines and the data is compressed by storing the spline parameters [12].

Review of some other time-domain ECG data compression methods can be found in Reference 5.

\subsection{Frequency-Domain Data Compression Methods}

Transform Coding (TC) is the most important frequency-domain digital waveform compression method [2]. The key is to divide the signal into frequency components and judiciously allocate bits in the frequency domain.

In most TC methods, the input signal is first divided into blocks of data and each block is linearly transformed into the "frequency" domain. Let $x=\left[x_{0}, x_{1}, \ldots, x_{N-1}\right]^{\mathrm{T}}$ be a vector obtained from a block of $N$ input samples. The transform domain coefficients, $v_{i}, i=0,1, \ldots, N-1$, are given by

$$
v=A x
$$

where $v=\left[v_{0} v_{1} \ldots v_{N-1}\right]^{\mathrm{T}}$, and $A$ is the $N \times N$ transform matrix representing the linear transform. A variety of transform matrices including the discrete Fourier transform matrix is used in digital waveform coding [2]. In this section discrete Karhunen-Loeve Transform (KLT) and Discrete Cosine Transform (DCT), which are the most used ones, are reviewed.

If the input signal is a wide sense stationary random process then the so-called optimum linear transform, KLT is well defined and it decorrelates the entries of the input vector. This is equivalent to removing all the unnecessary information contained in the vector $x$. Therefore, by coding the entries of the $v$ vector, only the useful information is retained. The entries of the $v$ vector are quantized and stored. Usually, different quantizers are used to quantize the entries of the $v$ vector. In general, more bits are allocated to those entries which have high energies compared to the ones with low energies.

The KLT is constructed from the eigenvectors of the autocovariance matrix of the input vector $x$. In most practical waveforms the statistics of the input vector change from block to block. Thus for each block a new KLT matrix must be constructed. This is computationally very expensive (in some practical cases a fixed KLT matrix is estimated and it is assumed to be constant for a reasonable amount of duration). Furthermore, there is no fast algorithm similar to the Fast Fourier Transform (FFT) to compute the KLT.

The Discrete Cosine Transform (DCT) [2] was developed by Ahmed et al., to approximate KLT when there is high correlation among the input samples, which is the case in many digital waveforms including speech, music, and biomedical signals.

The DCT $v=\left[v_{0}, v_{1}, \ldots, v_{N-1}\right]^{\mathrm{T}}$ of the vector $x$ is defined as follows:

$$
\begin{aligned}
& v_{0}=\frac{1}{\sqrt{N}} \sum_{n=0}^{N-1} x_{n} \\
& v_{k}=\sqrt{\frac{2}{N}} \sum_{n=0}^{N-1} x_{n} \cos \frac{(2 n+1) k \pi}{2 N} \quad k=1,2, \ldots,(N-1)
\end{aligned}
$$


where $v_{k}$ is the $k$ th DCT coefficient. The inverse discrete cosine transform (IDCT) of $v$ is given as follows:

$$
\begin{gathered}
x_{n}=\frac{1}{\sqrt{N}} v_{0}+\sqrt{\frac{2}{N}} \sum_{k=1}^{N-1} v_{k} \cos \frac{(2 n+1) k \pi}{2 N} \quad n=0,1,2, \ldots,(N-1) \\
\left(\frac{k \pi}{4}, \frac{(k+1) \pi}{4}\right)_{k=0,1,2,3}
\end{gathered}
$$

There exist fast algorithms, Order (Nlog N), to compute the DCT [2]. Thus, DCT can be implemented in a computationally efficient manner. Two recent image and video coding standards, JPEG and MPEG, use DCT as the main building block.

A CR of 3 to 1 for single channel ECG is reported by using DCT and KLT based coders [13]. For multi-lead systems, two-dimensional (2-D) transform based methods can be used. A CR of 12 to 1 was reported [14] for a three-lead system by using a 2-D KLT. Recently, Philips [15] developed a new transform by using time-warped polynomials and obtained a CR of 26 to 9 . In this case a DCT based coding procedure produced a CR of 24 to 3 .

Since signal recording conditions and noise levels vary from study to study, a thorough comparison of the coding methods is very difficult to make. But, frequency-domain coding methods produce higher coding results than time-domain coding methods.

\subsection{Wavelet or Subband Coding}

Wavelet Transform (WT) is a powerful time-frequency signal analysis tool and it is used in a wide variety of applications including signal and image coding [16]. WT and Subband Coding (SBC) are closely related to each other. In fact the fast implementation of WTs are carried out using Subband (SB) filter banks. Due to this reason WT based waveform coding methods are essentially similar to the SBC based methods.

In an SBC structure the basic building block is a digital filter bank which consists of a lowpass and a highpass filter. In the ideal case, the passband (stopband) of the lowpass filter is $[0, \pi / 2][\pi / 2, \pi]$. Let $\mathrm{H}_{l}\left(e^{j \omega}\right) \mathrm{H}_{u}\left(e^{j \omega}\right)$ be the frequency response of the lowpass (highpass) filter. In SBC the input signal which is sampled at the rate of $f_{s}$ is filtered by $\mathrm{H}_{l}\left(e^{j \omega}\right)$ and $\mathrm{H}_{u}\left(e^{j \omega}\right)$ and the filter outputs are downsampled by a factor of two (every other sample is dropped) in order to maintain the overall sampling rate to be equal to the original rate. In this way two subsignals, $x_{i}(n)$ and $x_{u}(n)$ which both have a sampling rate of $f_{s} / 2$ are obtained. The block diagram of a two-level SBC structure is shown in Figure 3.1.

The subsignal, $x_{i}(n)\left[x_{u}(n)\right]$, contains the lowpass (highpass) frequency domain information of the original signal. The subsignals, $x_{i}(n)$ and $x_{u}(n)$, can be further divided into their frequency components in a similar manner. This SB division process can be repeated until the frequency domain is divided in a sufficient manner. In Reference 17, the two-level subband structure of Figure 3.2 which divides the frequency domain into four regions, is used to code a single channel ECG signal. The resulting subsignals can be encoded by various coding methods including DPCM, Entropy coding, and transform coding which should be designed to exploit the special nature of the subsignals to achieve the best possible CR for each band. Recently, very successful special encoders which take advantage of the tree structure of the decomposition were developed for compressing the subsignals [18]. The signal reconstruction from the coded subsignals is carried out by using a filter bank consisting of two filters, $G_{l}\left(e^{j \omega}\right)$ and $G_{u}\left(e^{j \omega}\right)$. In this case the subsignal, $x_{i}(n)\left[x_{u}(n)\right]$ is first upsampled by inserting a zero between every other sample and filtered by $\mathrm{G}_{l}\left(e^{j \omega}\right)\left[\mathrm{G}_{u}\left(e^{j \omega}\right)\right]$. The reconstructed signal, $y(n)$, is the sum of the outputs of these two filters.

By properly selecting the filters, $\mathrm{H}_{l}(z), \mathrm{H}_{u}(z), \mathrm{G}_{l}(z)$, and $\mathrm{G}_{u}(z)$, perfect reconstruction is possible, that is,

$$
y(n)=x(n-K)
$$




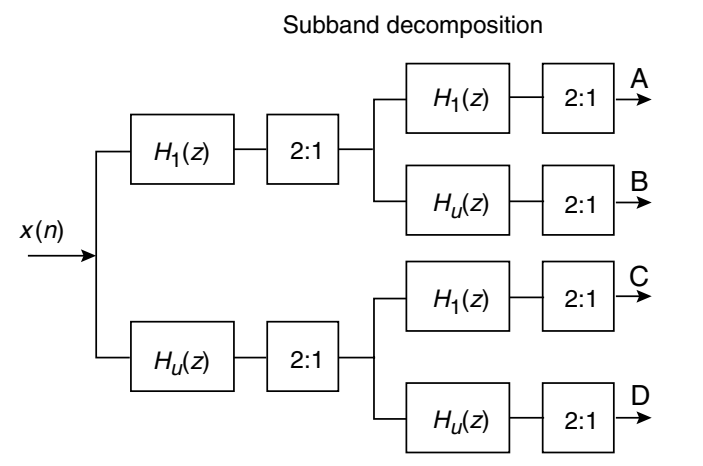

2:1: Downsampler by a factor of 2
Subband reconstruction

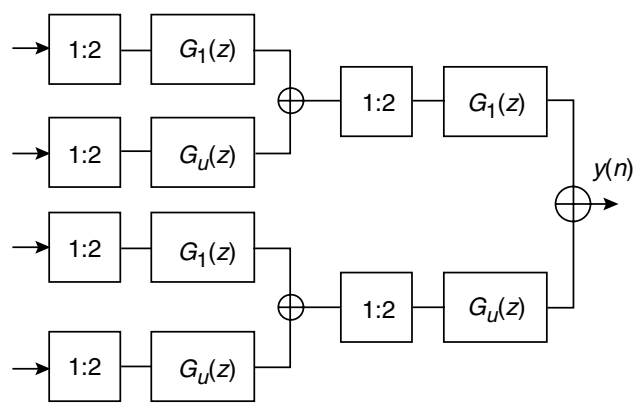

2:1: Upsampler by a factor of 2

FIGURE 3.2 Two-level (four branch) subband coder structure. This structure divides the frequency domain into four regions.

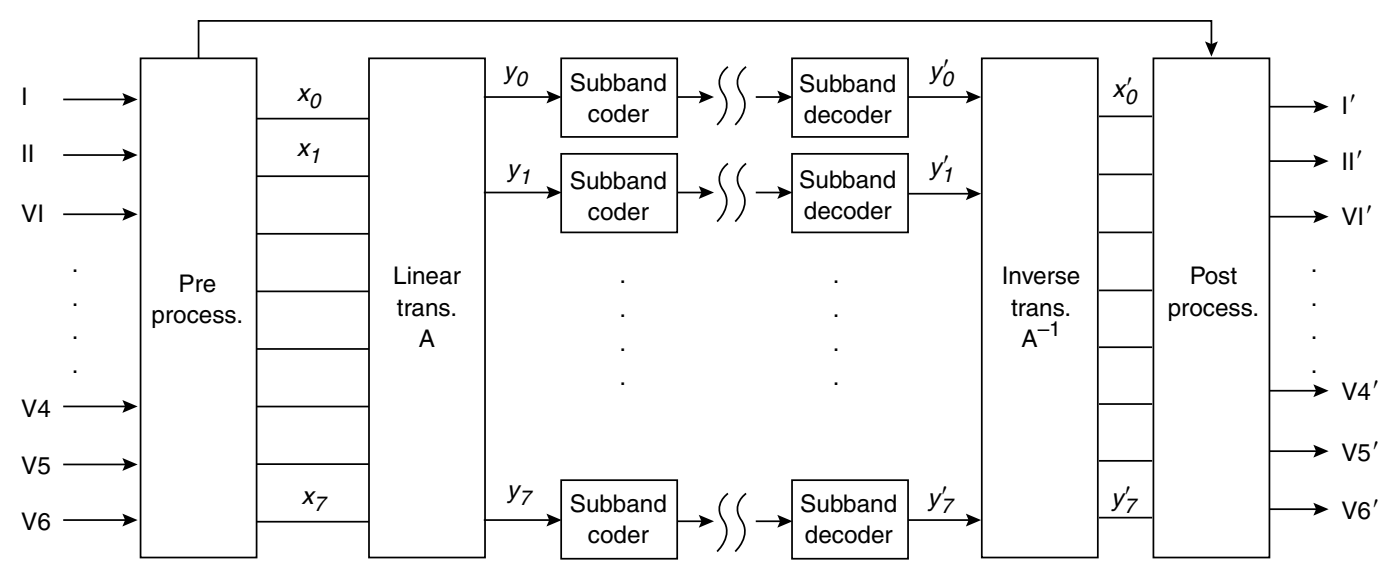

FIGURE 3.3 Block diagram of the multichannel ECG data compression scheme.

in the absence of quantization errors [16]. In other words, filtering and downsampling operations in the SB decomposition structure do not introduce any loss.

A CR 5.22 corresponding to a PRD of 5.94\% is reported for an ECG signal sampled at $500 \mathrm{~Hz}$ with 12 bit/sample resolution [17].

Other WT based ECG coding methods include [16,19-21].

\subsection{Hybrid Multichannel ECG Coding}

In this section, a hybrid frequency domain multichannel compression method [22] for the so-called standard lead [23] ECG recording system is described. The block diagram of this system is shown in Figure 3.3. The main idea is to exploit not only the correlation between the consecutive ECG samples, but also the inherent correlation among the ECG channels.

The standard lead system has 12 ECG channels. In this method, the recorded digital signals are first passed through a preprocessor. The function of the preprocessor is to prepare raw ECG data for further processing. After preprocessing the input signals, the resulting discrete-time sequences are linearly transformed into another set of sequences. The aim of this linear transformation is to decorrelate the highly correlated ECG lead signals. In a way, this idea is similar to representing the RGB color components of an image in terms of luminance and chrominance components. 
The transformation matrix, A, can be the matrix of the optimum transform, KLT, or the DCT matrix. Another approach is to use a nonlinear transform such as an Independent Component Analyzer (ICA) [24]. Lastly, to compress the transform domain signals, various coding schemes which exploit their special nature are utilized.

In the following sections, detailed descriptions of the sub-blocks of the multichannel ECG compression method are given.

\subsubsection{Preprocessor}

The standard lead ECG recording configuration consists of 12 ECG leads, I, II, III, AVR, AVL, AVF, V1, V2, .., V6. The leads, III, AVR, AVL, and AVF, are linearly related to I and II. Therefore, eight channels are enough to represent a standard 12-channel ECG recording system.

The preprocessor discards the redundant channels, III, AVR, AVL, and AVF, and rearranges the order of the ECG channels. The six precordial (chest) leads, V1, ., V6, represent variations of the electrical heart vector amplitude with respect to time from six different narrow angles. During a cardiac cycle it is natural to expect high correlation among precordial leads so the channels $\mathrm{V} 1, \ldots, \mathrm{V} 6$ are selected as the first 6 signals, that is, $x_{i-1}=V_{i}, i=1,2, \ldots, 6$. The two horizontal lead waveforms (I and II) which have relatively less energy contents with respect to precordial ECG lead waveforms are chosen as seventh, $x_{6}=\mathrm{I}$, and eighth channels, $x_{7}=\mathrm{II}$. A typical set of standard ECG lead waveforms, $x_{i}, i=0,1, \ldots, 7$, are shown in Figure 3.4.

The aim of the reordering the ECG channels is to increase the efficiency of the linear transformation operation which is described in the next section.

\subsubsection{Linear Transformer}

The outputs of the preprocessor block, $x_{i}, i=0,1, \ldots, 7$, are fed to the linear transformer. In this block, the ECG channels are linearly transformed to another domain, and eight new transform domain signals $y_{i}$, $i=0,1, \ldots, 7$, are obtained which are significantly less correlated (ideally uncorrelated) than the ECG signal set, $x_{i}, i=0,1, \ldots, 7$. The transform domain samples at discrete-time instant $m$ are given as follows:

$$
Y_{m}=A \cdot X_{m}
$$

where $Y_{m}=\left[y_{0}(m), \ldots, y_{N-1}(m)\right] T, X_{m}=\left[x_{0}(m), \ldots, x_{n-1}(m)\right] T$, and $A$ is the $N \times N$ transform matrix.

The optimum linear transform, discrete KLT, can be properly defined for stationary random processes and the entries of the tranform matrix, $A_{K L T}$ depending on the statistics of the random processes. For slowly varying, unstationary signals, an approximate KLT matrix can also be defined. Although ECG signals cannot be considered to be wide-sense stationary-random processes, a covariance matrix, $\hat{C}_{x}$, of the ECG channels is estimated as follows:

$$
\hat{C}_{x}=\frac{1}{M} \sum_{i=0}^{M-1}\left[\begin{array}{c}
x_{0}(i) \\
\vdots \\
x_{N-1}(i)
\end{array}\right]\left[x_{0}(i) \cdots x_{N-1}(i)\right]
$$

where $N$ is the number of the ECG channels and $\mathrm{M}$ is the number of ECG samples per channel used. The $N \times N$ ECG channel covariance matrix, $\hat{C}_{x}$, is used in the construction of an approximate KLT matrix. Rows of the approximate KLT matrix are the eigenvectors of $\hat{C}_{x}$. Typical approximate KLT matrices can be found in [22].

Although there is no fast algorithm to compute the KL transform, the computational burden is not high because $N=8$. The DCT can also be used as a linear transformer because it approximates the KLT. 

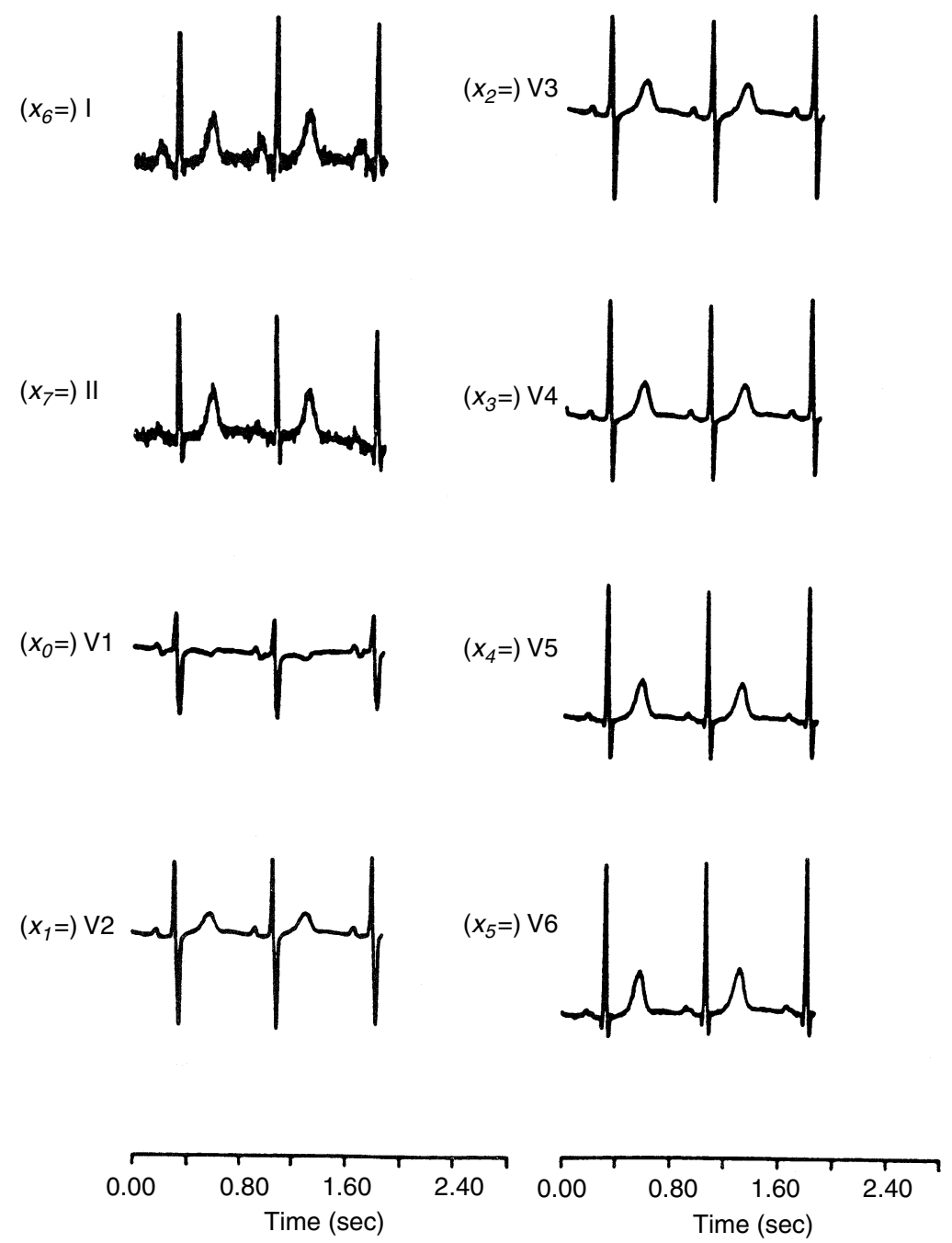

FIGURE 3.4 A typical set of standard ECG lead waveforms $x_{i}, i=0,1, \ldots, 7$.

\subsubsection{Compression of the Transform Domain Signals}

In this section the compression of the uncorrelated transform domain signals $y_{k}, k=0,1, \ldots, 7$, are described. In Figure 3.5 a typical set of uncorrelated signals, $y_{k}, k=0,1, \ldots, 7$, are shown. The signals in Figure 3.5 are obtained by KL transforming the ECG signals, $x_{k}, k=0,1, \ldots, 7$, shown in Figure 3.4.

Transform domain signals, $y_{k}, k=0,1, \ldots, 7$, are divided into two classes according to their energy contents. The first class of signals, $y_{0}, y_{1}, \ldots, y_{4}$, have higher energy than the second class of signals, $y_{5}$, $y_{6}$, and $y_{7}$. More bits are allocated to the high energy signals, $y_{0}, y_{1}, \ldots, y_{4}$, compared to the low energy signals, $y_{5}, y_{6}$, and $y_{7}$ in coding.

\subsubsection{Subband Coder (SBC)}

Higher energy signals, $y_{0}(n), y_{1}(n), \ldots, y_{4}(n)$, contain more information than the low energy signals, $y_{5}(n), y_{6}(n)$, and $y_{7}(n)$. Therefore, the high energy signals, $y_{0}(n), \ldots, y_{4}(n)$ should be compressed very accurately. The signals, $y_{0}(n), \ldots, y_{4}(n)$, are compressed using the SBC [17] because this coding scheme does not introduce any visual degradation as pointed out in the previous section [9]. 


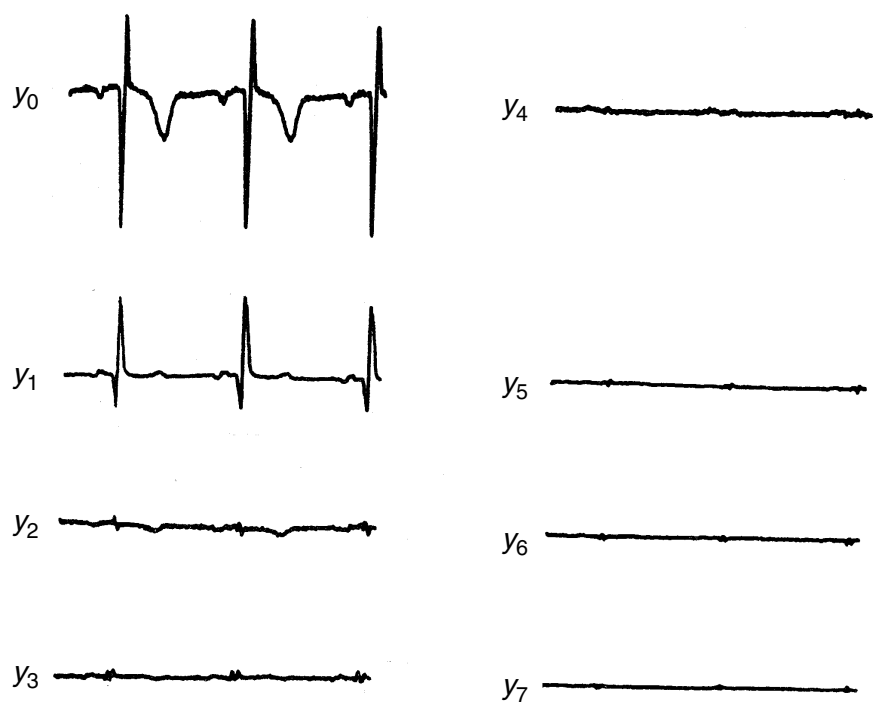

FIGURE 3.5 Uncorrelated signals $y_{i}, i=0,1,2, \ldots, 7$, corresponding to the ECG signals shown in Figure 3.4.

Each signal $y_{i}$ is decomposed into four subsignals by using a filter bank in a tree-structured manner. In this way the signal $y_{i}$ is divided into four consecutive bands, $[l \pi / 4,(l+1) \pi / 4], l=0,1,2,3$, in the frequency domain [2]. For example, $y_{i 00}$ (the subsignal at branch A of Figure 3.2) comes from the lowpass frequency band, $[0, \pi / 4]$, of the signal $y_{i}$. In the coding of the subband signals, $y_{i j k}, j=0,1 ; k=0,1$, the advantage is taken of the nonuniform distribution of energy in the frequency domain to judiciously allocate the bits. The number of bits used to encode each frequency band can be different, so the encoding accuracy is always maintained at the required frequency bands [2].

It is observed that the energy of the signal $y_{i}$ is mainly concentrated in the lowest frequency band $[0, \pi / 4]$. Because of this, the lowband subsignal $y_{i, 0,0}$ has to be carefully coded. High correlation among neighboring samples of $y_{i, 0,0}$ makes this signal a good candidate for efficient predictive or transform coding. The subsignals $y_{i, 0,0}$ are compressed using a DCT based scheme. After the application of DCT with a block size of 64 samples to the lowband subsignal, $y_{i, 0,0}$, the transform domain coefficients, $g_{i, 0,0}(k)$, $k=0,1, \ldots, 63$ are obtained and they are thresholded and quantized. The coefficients whose magnitudes are above a preselected threshold, $\mathrm{b}$, are retained and the other coefficients are discarded. Thresholded DCT coefficients are quantized for bit level representation. Thresholded and quantized nonzero coefficients are variable-length coded by using an amplitude table and the zero values are runlength coded. The amplitude and runlength lookup tables are Huffman coding tables which are obtained according to the histograms of the DCT coefficients [22].

In practice, ECG recording levels do not change from one recording to another. If drastic variations occur, first scale the input by an appropriate factor, then apply DCT.

Bandpass and highpass subsignals, $y_{i, 0,1}, y_{i, 1,0}, y_{i, 1,1}$ (branches $\mathrm{B}, \mathrm{C}$, and $\mathrm{D}$ ), are coded using nonuniform quantizers. After quantization, a code assignment procedure is realized using variable length amplitude and runlength lookup tables for zero values. The look up tables are obtained according to the histograms of quantized subband signals.

The bit streams which are obtained from coding of four subband signals are multiplexed and stored. Appropriate decoders are assigned to each branch to convert the bit streams into time domain samples and the four-branch synthesis filter bank performs the reconstruction [17].

The low energy signals, $y_{5}(n), y_{6}(n), y_{7}(n)$, are also coded by using the SB coder previously explained. However, it is observed that all the subsignals except the subsignal at branch A of Figure 3.2 contain very little information when subband decomposition is applied to the signals $y_{5}(n), y_{6}(n), y_{7}(n)$. Due to this 

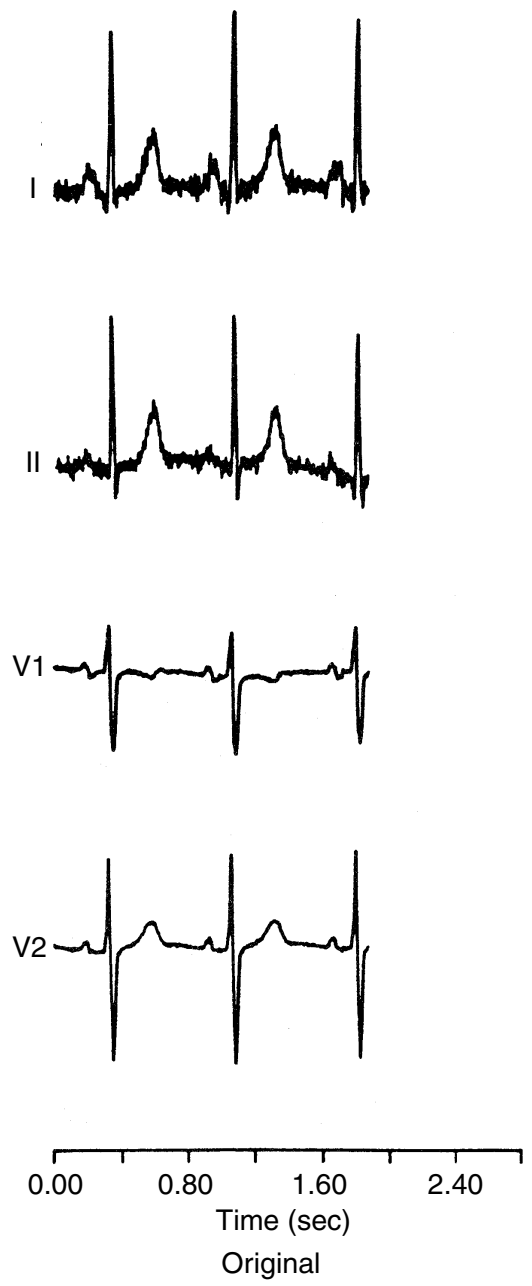

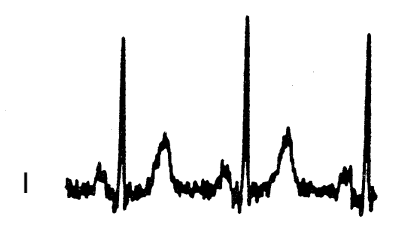

II
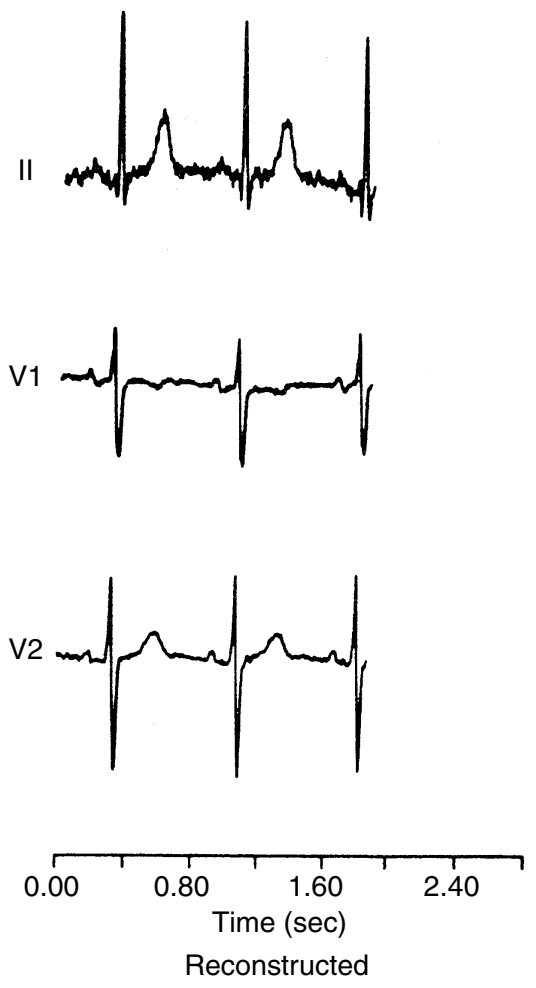

FIGURE 3.6 The original and reconstructed ECG lead signals I, II, V1, and V2 (CR = 6.17, APRD = 6).

fact, only the subsignals at branch A (lowband signals) are processed. The other branches which have very little energy are discarded.

Original and reconstructed ECG waveforms are shown in Figure 3.6 for $\mathrm{CR}=6.17(\mathrm{CR}=7.98)$ with $\mathrm{PRD}=6.19 \%$ when DCT (KLT) is used as the Linear Transformer. Recorded ECG signals are sampled at $500 \mathrm{~Hz}$ with $12 \mathrm{bit} / \mathrm{sample}$ resolution. Also, the raw ECG signals are filtered to attenuate the high frequency noise with a 33-tap equiripple Parks-McClellan FIR filter whose cutoff frequency is equal to $125 \mathrm{~Hz}$. In this case a $C R=9.41$ is obtained for a $\mathrm{PRD}=5.94 \%$.

The effect of compressing the data on diagnostic computer analysis results is tested on Cardionics program, which is derived from the Mount Sinai program developed by Pordy et al., in conjunction with CRO-MED Bionics Company [25]. Morphological measurements include (1) intervals — PR, QRS, QT, (2) “width"s - Q, R, S, R', S', T, P, (3) amplitudes - P, Q, R, R', S, S', T, JJ, and (4) areas of QRS and QRST. There was no difference in the measurement results of both the compressed and the original data.

It is experimentally observed that the multichannel technique produces better compression results than single channel schemes. Also, the computational complexity of the multichannel scheme is comparable to single channel ECG coding schemes and the algorithm can be implemented by using digital signal processor for real-time applications, such as transmission of ECG signals over telephone lines. 


\subsection{Conclusion}

In this section biomedical signal compression methods are reviewed. Most of the biomedical data compression methods have been developed for ECG signals. However, these methods can be applied to other biomedical signals with some modifications.

It is difficult to compare the efficiency of biomedical data compression schemes because coding results of various data compression schemes are obtained under different recording conditions such as (1) sampling frequency, (2) bandwidth, (3) sample precision, and (4) noise level, which may drastically affect the currently used performance measures.

\section{References}

[1] Willems, J. “Common standards for quantitative electrocardiography," J. Med. Eng. Techn., 9, 209217, 1985.

[2] Jayant, N.S. and Noll, P. Digital Coding of Waveforms, Englewood Cliffs, NJ, Prentice-Hall, 1984.

[3] Ruttiman, U.E. and Pipberger, H.V. "Compression of the ECG by prediction or interpolation and entropy coding," IEEE Trans. Biomed. Eng., 26, 613-623, 1979.

[4] Cox J.R. et al., "AZTEC: a preprocessing program for real-time ecg rhythm analysis," IEEE Trans. Biomed. Eng., 15, 128-129, 1968.

[5] Jalaleddine, S.M.S., Hutchens, C.G., Strattan, R.D., and Coberly, W.A. "ECG data compression techniques: a unified approach,” IEEE Trans. Biomed. Eng., BME-37, 329-343, 1990.

[6] Abenstein, J.P. and Tompkins, W.J. "New data reduction algorithm for real-time ECG analysis," IEEE Trans. Biomed. Eng., 29, 43-48, 1982.

[7] Mueller, W.C. "Arrhythmia detection program for an ambulatory ECG monitor," Biomed. Sci. Instrument, 14, 81-85, 1978.

[8] Imai, H., Kimura, N., and Yoshida, Y. "An efficient encoding method for ECG using spline functions," Syst. Comput, Jpn, 16, 85-94, 1985.

[9] Jalaleddine, S.M.S., Hutchens, C.G., Strattan, R.D., and Coberly, W.A. "Compression of Holter ECG Data," Biomed. Sci. Instrum., 24, 35-45, 1988.

[10] Hamilton, P.S. and Tompkins, W.J. "Compression of the ambulatory ECG by average beat subtraction and residual differencing," IEEE Trans. Biomed. Eng., 38, 253-260, 1991.

[11] Angelidou, A. et al., "On AR modeling for MEG spectral estimation, data compression, and classification," Comput. Biol Med., 22, 379-387, 1992.

[12] Karczewicz, M. and Gabbouj, M. "ECG data compression by spline approximation," Signal Process., 59, 43-59, 1997.

[13] Ahmed, N., Milne, P.J., and Harris, S.G. "Electrocardiographic data compression via orthogonal transforms," IEEE Trans. Biomed. Eng., 22, 484-487, 1975.

[14] Womble, M.E. et al. "Data compression for storing and transmitting ECGs/VCGs," Proc. IEEE, 65, 702-706, 1977.

[15] Philips, W. "ECG data compression with time-warped polynomials," IEEE Trans. Biomed. Eng., 40, 1095-1101, 1993.

[16] Strang, G. and Nguyen, T. Wavelets and Filter Banks, Wellesley-Cambridge Press, MA, 1996.

[17] Aydin, M.C., Çetin, A.E., Köymen, H. "ECG data compression by sub-band coding," Electron. Lett., 27, 359-360, 1991.

[18] Shapiro, J.M. "Embedded image coding using zerotrees of wavelet coefficients," IEEE Trans. SP, 41, 3445-3462, 1993.

[19] Crowe, J.A. et al., "Wavelet transform as a potential tool for ECG analysis and compression," J. Biomed. Eng., 14, 268-272, 1992.

[20] Tai, S.C. "6-band subband coder on ECG waveforms," Med. Biolog. Eng. Comput., 30, 187-192, 1992. 
[21] Çetin, A.E., Tewfik, A.H. and Yardimci, Y. "ECG coding by wavelet transform extrema," IEEE Symp. Time-Freq. and Time-Scale, Philadelphia, 1994.

[22] Çetin, A.E., Köymen, H., and Aydn, M.C. "Multichannel ECG data compression by multirate signal processing and transform coding techniques," IEEE Trans. Biomed. Eng., 40, 495-499, 1993.

[23] Wyngaarden, J.B. and Smith, L.H. Textbook of Medicine, W.B. Saunders, Toronto, 1985.

[24] Vigario, R. and Oja, E. “ICA fixed point algorithm in extraction of artifacts from EEG," NORSIG'96, Finland, pp. 383-386, 1996.

[25] Pordy, L., Jaffe, H., Chelsky, K., Freiedberg, C.K., Fullowes, L., and Bonner, R.E. “Computer diagnosis of electrodcardiograms: a computer program for contour analysis with classical results of rhythm and contour interpretation," Comput. Biomed. Res., 1, 408-433, 1968.

[26] Bertrand, M., Guardo, R., Roberge, F.A., and Blondeau, P. "Microprocessor application for numerical ECG encoding and transmission," Proc. IEEE, 65, 714-722, 1977.

[27] Ider, Y.Z. and Köymen, H."A new technique for line interference monitoring and reduction in biopotential amplifiers," IEEE Trans. Biomed. Eng., 37, 624-631, 1990.

\section{Further Information}

Biomedical signal compression is a current research area and most of the research articles describing the advances in biomedical data compression appear in the following journals; IEEE Transactions on Biomedical Engineering and Signal Processing, Journal of Biomedical Engineering, and Medical and Biological Engineering and Computing. 